\title{
S6K1 regulates hematopoietic stem cell self-renewal and leukemia maintenance
}

\author{
Joydeep Ghosh,, ${ }^{1,2}$ Michihiro Kobayashi, ${ }^{2}$ Baskar Ramdas, ${ }^{2}$ Anindya Chatterjee, ${ }^{2}$ Peilin Ma, ${ }^{2}$ Raghuveer Singh Mali, ${ }^{2}$ \\ Nadia Carlesso, ${ }^{2}$ Yan Liu, ${ }^{2}$ David R. Plas, ${ }^{3}$ Rebecca J. Chan, ${ }^{2}$ and Reuben Kapur ${ }^{1,2}$ \\ 'Department of Microbiology and Immunology and 2Department of Pediatrics, Herman B. Wells Center for Pediatric Research, Indiana University School of Medicine, Indianapolis, Indiana, USA. \\ ${ }^{3}$ University of Cincinnati College of Medicine, Cincinnati, Ohio, USA.
}

\begin{abstract}
Hyperactivation of the mTOR pathway impairs hematopoietic stem cell (HSC) functions and promotes leukemogenesis. mTORC1 and mTORC2 differentially control normal and leukemic stem cell functions. mTORC1 regulates p70 ribosomal protein S6 kinase 1 (S6K1) and eukaryotic initiation factor 4E-binding (elF4E-binding) protein 1 (4E-BP1), and mTORC2 modulates AKT activation. Given the extensive crosstalk that occurs between mTORC1 and mTORC2 signaling pathways, we assessed the role of the mTORC1 substrate S6K1 in the regulation of both normal HSC functions and in leukemogenesis driven by the mixed lineage leukemia (MLL) fusion oncogene MLL-AF9. We demonstrated that S6K1 deficiency impairs self-renewal of murine HSCs by reducing p21 expression. Loss of S6K1 also improved survival in mice transplanted with MLL-AF9-positive leukemic stem cells by modulating AKT and 4E-BP1 phosphorylation. Taken together, these results suggest that S6K1 acts through multiple targets of the mTOR pathway to promote self-renewal and leukemia progression. Given the recent interest in S6K1 as a potential therapeutic target in cancer, our results further support targeting this molecule as a potential strategy for treatment of myeloid malignancies.
\end{abstract}

\section{Introduction}

Aberrant regulation of the intracellular signaling pathways that underlie normal hematopoietic stem cell (HSC) renewal and differentiation is one of the pathogenic hallmarks of human leukemia. mTOR plays an essential role in regulating HSC generation and is involved in leukemogenesis (1-3). mTOR is present in 2 distinct multiprotein complexes - mTORC1 and mTORC2 (4). Among the various substrates phosphorylated by mTORC1, S6 kinase 1 (S6K1) and eukaryotic initiation factor 4E-binding (eIF4E-binding) protein 1 (4E-BP1) are perhaps the best-characterized downstream substrates. Given the known crosstalk between mTORC1 and mTORC2, the specific role of the individual downstream substrates of mTORC1 and MTORC2 in regulating benign and malignant hematopoiesis can best be assessed by examining HSCs lacking the downstream components of this pathway. Here, we investigated the role of S6K1 in steady-state, stress, and malignant hematopoiesis using a mouse model of S6K1 deficiency. Our studies reveal that the self-renewal of HSCs and the leukemic potential of these cells are similarly dependent on S6K1 and provide mechanistic insight into how loss of S6K1 may contribute to normal and malignant stem cell function or functions and establish S6K1 as a potential therapeutic target in the treatment of leukemia.

\section{Results and Discussion}

We first examined the expression of $S 6 k 1$ in BM-derived fractions of HSCs and progenitor cells (HSC/Ps). S6k1 is expressed in long-term HSCs (LT-HSCs) as well as in various progenitor

Conflict of interest: The authors have declared that no conflict of interest exists. Submitted: September 4, 2015; Accepted: April 27, 2016.

Reference information: J Clin Invest. 2016;126(7):2621-2625. doi:10.1172/JCI84565. populations (Figure 1A). However, the expression of $S 6 k 1$ is significantly higher in granulocyte-macrophage progenitors (GMPs) and megakaryocyte-erythroid progenitors (MEPs) and significantly less in mature myeloid cells relative to LT-HSCs (Figure 1A). To address the role of S6K1 in steady-state hematopoiesis, we examined the effect of S6K1 deficiency on BM-derived HSC/ Ps. S6K1 deficiency resulted in a significant reduction in $\mathrm{BM}$ mononuclear cells (MNCs) (Figure 1B). Although S6K1 deficiency does not affect HSC frequency, a decrease in the absolute number of LT-HSCs (CD150 $\left.{ }^{+} \mathrm{CD} 48^{-} \mathrm{LSK}\right)$ and MPPs (CD150 CD48-LSK) was observed (Figure 1C and Supplemental Figure 1, $\mathrm{A}$ and B; supplemental material available online with this article; doi:10.1172/JCI84565DS1). The frequency of MPPs in $S 6 k 1^{-/-} \mathrm{BM}$ was reduced, while the frequency of hematopoietic progenitor cells 1 (HPC1) (CD150-CD48 $\left.{ }^{+} \mathrm{LSK}\right)$ and $\mathrm{HPC} 2\left(\mathrm{CD} 150^{+} \mathrm{CD} 48^{-}\right.$ LSK) was increased (Supplemental Figure $1, \mathrm{~A}^{-} \mathrm{C}$ ). The frequency of GMPs was increased in $S 6 \mathrm{k1}^{-/}$mice, while the frequency of MEPs was reduced (Figure 1D and Supplemental Figure 1D). The absolute number of common myeloid progenitors (CMPs) and MEPs was significantly reduced following S6K1 deficiency (Figure 1D). The frequency of myeloid cells in the BM of $S 6 k 1^{-/-}$mice was also elevated, while the frequency and absolute number of $\mathrm{B}$ cells were reduced (Supplemental Figure 1, E-G). The changes in $\mathrm{BM}$ cellularity as well as in the GMP and B cell fractions observed in $S 6 \mathrm{k1}^{-/-}$mice are similar to those reported in mTORC1-deficient mice $(2,3)$. Given that mTORC1 regulates quiescence in HSCs (5), we analyzed the impact of S6K1 deficiency on HSC quiescence by assessing the level of expression of Ki-67, a marker absent in quiescent cells. S6K1-deficient LT-HSCs showed reduced frequency of Ki-67-negative cells, suggesting decreased quiescence in LT-HSCs (Figure 1E), which was associated with a reduction in 
A

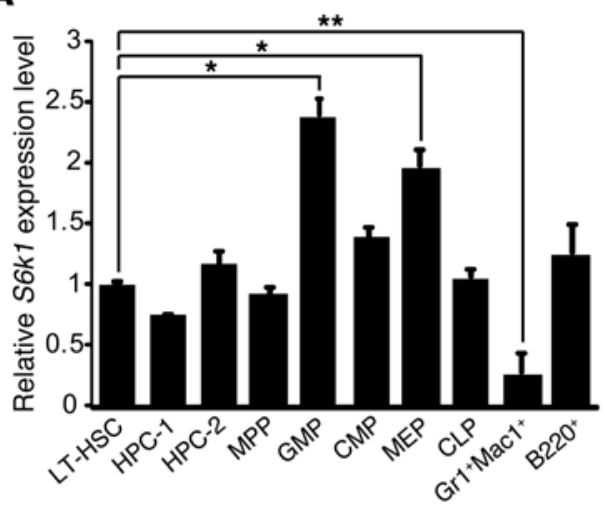

B

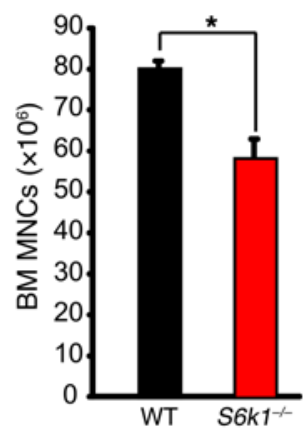

C

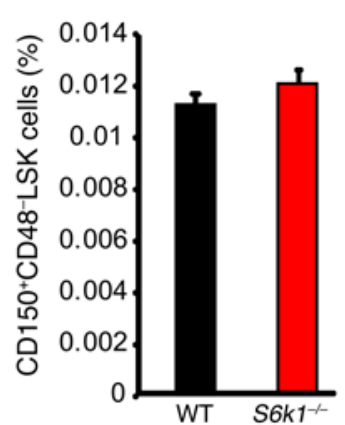

Absolute no.

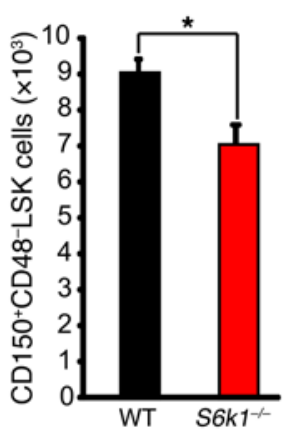

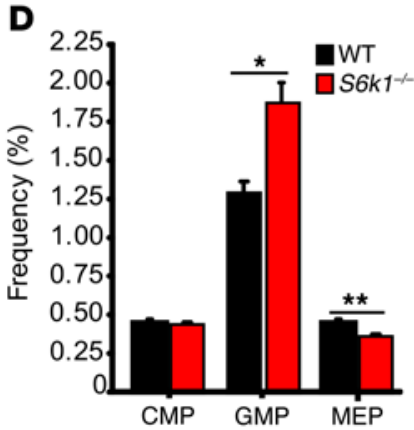

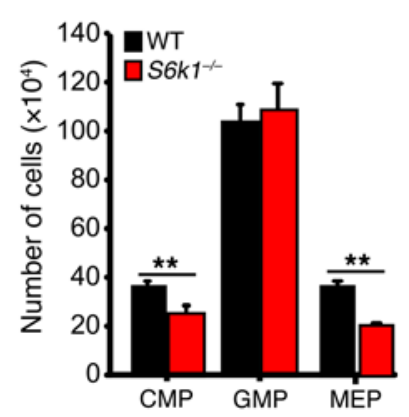

E

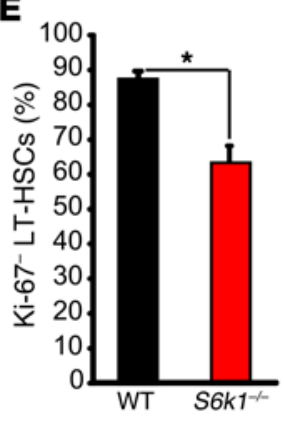

$\mathbf{F}$

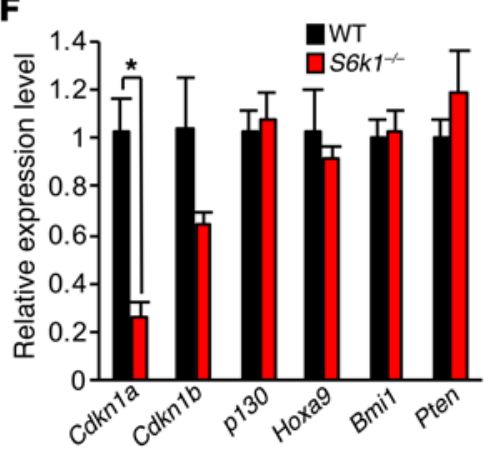

Figure 1. S6K1 regulates HSC/Ps frequencies and absolute numbers in the BM. (A) Relative expression level of S6k1 in purified BM-derived hematopoietic subsets as assessed by quantitative real-time reverse-transcription PCR (qRT-PCR). Data are from a representative experiment performed twice independently in replicates of 4 ; mean \pm SD. ${ }^{*} P<0.001 ;{ }^{* *} P<0.0001$. (B) Total BM MNCs derived from WT and S6k1 ${ }^{-1-}$ mice; mean \pm SEM; $n=4-5 /$ group. ${ }^{*} P<0.002, t$ test. Data representative of 3 independent experiments. (C) Frequency and absolute number of LT-HSCs in the BM of WT and S6k1/- mice. $n=4-5$ /group; mean \pm SEM. ${ }^{*} P<0.02, t$ test. (D) Frequency and absolute number of hematopoietic progenitor subpopulations in bone marrow of WT and S6 k $1^{-1-}$ mice; $n=4-5 /$ group; mean \pm SEM. ${ }^{*} P<0.02 ;{ }^{* *} P<0.005, t$ test. (E) Frequency of Ki-67-negative LT-HSCs in the BM of WT and S6k $1^{1 /-}$ mice; mean \pm SEM. ${ }^{*} P<0.01, t$ test. $n=4-5$ mice/group. (F) Expression levels of genes regulating quiescence in LT-HSCs of WT and S6k $1^{1 /-}$ mice; mean \pm SEM. ${ }^{*} P<0.005$. Data are representative of 2 independent experiments performed in replicates of 3 .

the expression of cyclin-dependent kinase inhibitor 1A (Cdkn1a) encoding p21 (Figure 1F).

Given the impaired quiescence and the associated decline in the expression of Cdkn1a due to S6K1 deficiency in LT-HSCs, we hypothesized that under conditions of stress, reduced quiescence might result in enhanced HSC depletion and hematopoietic failure in $S 6 \mathrm{k1}^{-1-}$ mice. To test this, we treated WT and $S 6 \mathrm{k1}^{-1-}$ mice with a single dose of 5 -fluorouracil (5-FU) to examine the recovery of HSCs. The BM cellularity in $S 6 \mathrm{k1}^{-/-}$mice remained low only on the fifteenth day after 5-FU treatment compared with that in WT (Supplemental Figure 2A). However, the frequency of LSK cells in $\mathrm{S}_{\mathrm{k}} \mathrm{k1}^{-/-}$mice was higher than that in WT mice on the ninth day after 5-FU injection (Figure 2A and Supplemental Figure 2B). S6K1-deficient LSK cells also demonstrated increased cycling following myelosuppression (Figure $2 \mathrm{~B}$ and Supplemental Figure $2 \mathrm{C}$ ), which was uniquely associated with increased expression of cyclin G1 (Ccng1), a protein highly expressed in self-renewing HSCs (ref. 6, Figure 2C, and Supplemental Figure 2D). Given these observations, we hypothesized that $S 6 \mathrm{k1}^{-/-}$HSCs are likely to be more susceptible to repeated 5-FU stress (7). To assess this in detail, WT and $S 6 \mathrm{k1}^{-/-}$mice were subjected to weekly doses of 5 -FU. Most of the WT mice survived until the 20th day after the first 5 -FU injection, and approximately $20 \%$ of these mice sur- vived beyond 4 weeks after injection. In contrast, all $S 6 k 1^{-/-}$mice died by day 18 after the first injection of 5-FU (Figure 2D). To determine whether the susceptibility of $S 6 \mathrm{k1}^{-/-}$mice to repeated stress is due to an HSC-specific defect, we transplanted WT mice with either WT or $S 6 \mathrm{k1}^{-/-} \mathrm{HSC} / \mathrm{Ps}$ and treated the recipients with weekly doses of 5-FU. WT mice transplanted with $S 6 \mathrm{k1}^{-/-}$ HSC/Ps showed significantly reduced median survival compared with WT mice transplanted with WT HSC/Ps (Figure 2E). 5-FUtreated HSC/Ps also showed reduced engraftment following S6K1 deficiency at increased cell dilution, but not in lower dilution (Figure 2F and Supplemental Figure 2E). Thus, although S6K1 deficiency causes an increase in HSC frequency upon 5-FU administration, it also results in the loss of quiescence and resultant failure in HSC regeneration following repeated myelosuppressive stress. Overall, our results show that S6K1 regulates quiescence following regeneration of HSCs after myeloablative stress and that deficiency of S6K1 renders HSCs susceptible to repeated stress and reduced engraftment potential.

As S6K1 deficiency results in reduced number and quiescence of phenotypically defined LT-HSCs, we assessed whether S6K1 regulates the function of HSCs. We performed competitive transplants using WT or $S 6 k 1^{-/-}$BM MNCs along with competitor cells. Initially, we observed a defect in the short-term engraftment in 
A

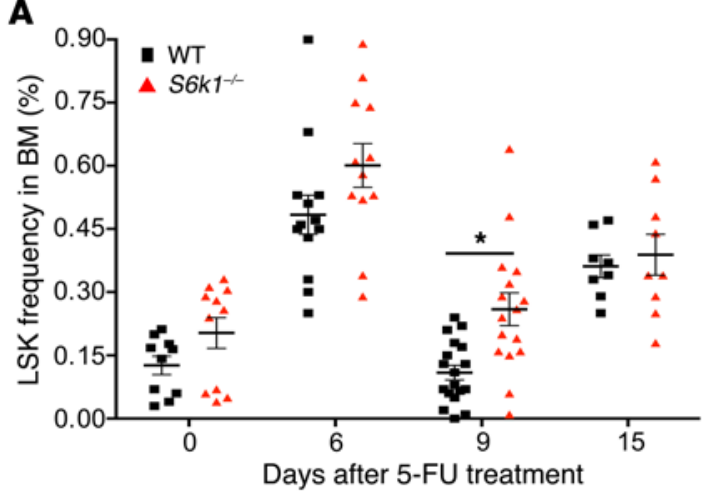

B

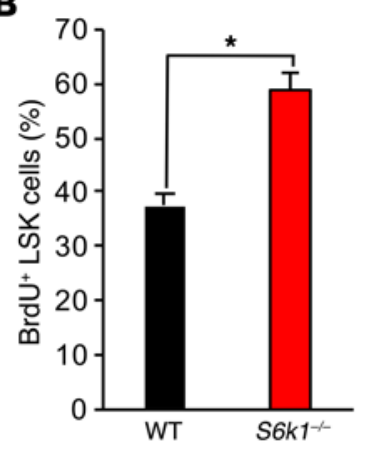

C

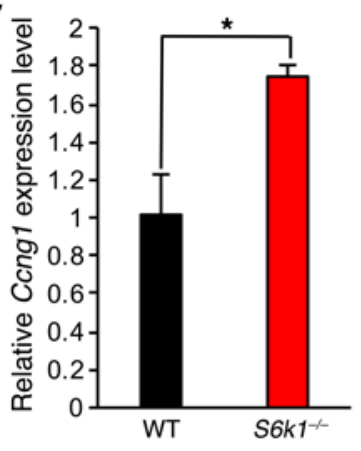

D

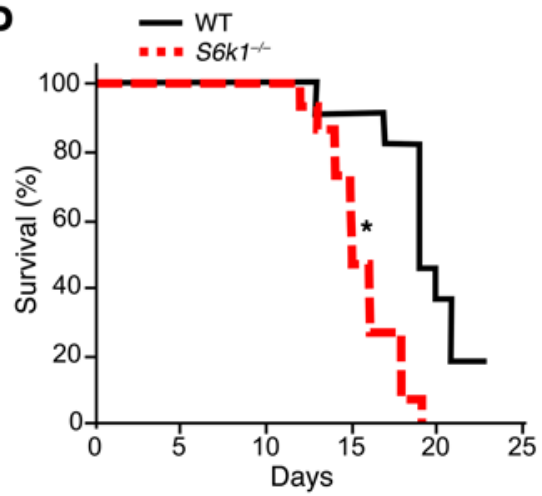

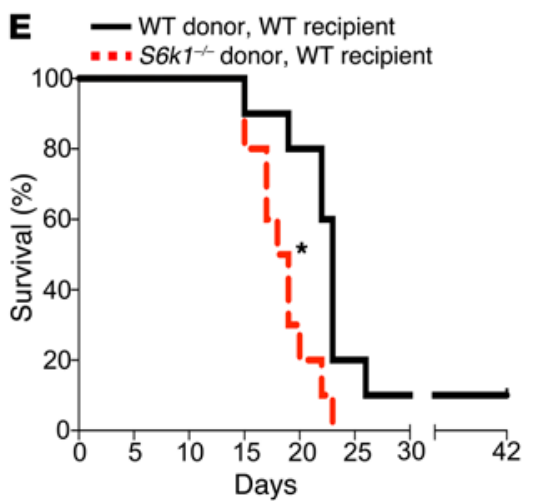

$\mathbf{F}$

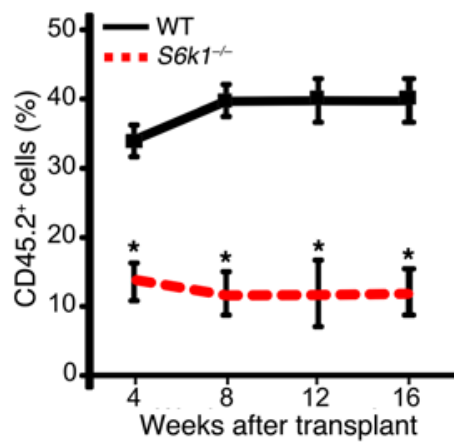

Figure 2. S6K1 regulates quiescence of HSCs following regeneration from myeloablative stress. (A) BM LSK frequency in WT and S6k $1^{-1-}$ mice following a single dose of 5-FU; mean \pm SEM. ${ }^{*} P<0.03, t$ test. $n=8-18 /$ group. (B) Quantitative representation of BrdU ${ }^{+}$LSK cells in the BM of WT and S6k1/- mice on the ninth day after 5 -FU treatment; mean \pm SEM. ${ }^{*} P<0.05, t$ test. $n=3$ /group. Data are representative of 2 independent experiments. (C) Expression levels of Ccng1 in BM LSK cells of WT and S6k1-/- mice on ninth day after 5-FU treatment. Data are from a representative experiment performed twice independently. Experiments performed in quadruplicates; mean $\pm \mathrm{SD} .{ }^{*} \mathrm{P}<0.01$. (D) Kaplan-Meier survival curve of WT and $S 6 \mathrm{k} 1^{-1-}$ mice treated with weekly doses of 5-FU. ${ }^{*} P<0.001 . n=11-15 /$ group. (E) Kaplan-Meier survival curve of WT mice transplanted with WT and S6k $1^{-/-}$HSC/Ps and treated with weekly doses of 5-FU. ${ }^{*} P<0.007 . n=10$ /group. (F) WT and $56 \mathrm{k}^{-1-}$ mice were treated with a single dose of 5-FU. After 6 days, MNCs from treated mice were transplanted into lethally irradiated recipients at a dilution of 1:8. Quantitative representation of donor-derived cells in PB of recipients is shown; mean \pm SEM. $n=3$ /group. ${ }^{*} P<0.05,1$-way ANOVA.

recipient mice transplanted with $S 6 k 1^{-/-}$MNCs; however, the difference did not persist long term (Supplemental Figure 3, A and B). In contrast, following secondary transplantation, $\mathrm{S}_{6 \mathrm{k1}} \mathrm{1}^{-/} \mathrm{MNCs}$ showed a persistent reduction in the engraftment relative to WT controls (Supplemental Figure 3, C and D). To assess whether the self-renewal defect was HSC specific, we performed competitive transplants using sorted LT-HSCs from WT and S6 $\mathrm{k1}^{-/-}$mice. In the primary recipients, there was no difference in the engraftment of either WT or $\mathrm{S}_{61^{-/-}}$donor-derived cells (Supplemental Figure $3, \mathrm{E}$ and $\mathrm{F}$ ). There was also no difference in the differentiation of myeloid cells, B cells, or T cells at 24 weeks after transplantation between 2 groups (Supplemental Figure 3G). However, following secondary transplant with cells derived from the BM of primary recipients, $S 6 \mathrm{k1}^{-/-}$donor cells demonstrated a significantly reduced engraftment in the peripheral blood $(\mathrm{PB})$ of secondary recipients (Figure 3A and Supplemental Figure 3H). We further performed tertiary transplant with cells derived from the BM of secondary recipients. Recipients of $\mathrm{S}_{6} \mathrm{k1}^{-/-} \mathrm{HSCs}$ demonstrated reduced engraftment in the $\mathrm{PB}$ of tertiary recipients (Figure $3 \mathrm{~B}$ and Supplemental Figure 3I). The engraftment of $S 6 \mathrm{k1}^{-/-}$donor cells was significantly reduced in the BM of secondary recipients, while there was no difference in the engraftment between the 2 groups in primary transplant recipients (Supplemental Figure 3J). Consistent with our earlier observations (Figure 1F), Cdkn1a expression was reduced in S6K1-deficient LSK cells derived from the $\mathrm{BM}$ of secondary recipients relative to controls (Figure $3 \mathrm{C}$ and Supplemental Figure 3K). These results demonstrate that loss of S6K1 expression results in impaired self-renewal of HSCs, which is associated with reduced expression of $C d k n 1 a$.

Previous studies have shown that the mTORC1 pathway is hyperactivated in acute myeloid leukemias (AMLs) bearing the mixed lineage leukemia $(M L L)$ translocation and is associated with poor overall prognosis, including reduced survival $(8,9)$. Primary AML cells derived from patients show increased activation of S6K1 (10). We therefore assessed the role of S6K1 in the initiation and self-renewal of MLL-AF9-driven leukemogenesis. We transplanted WT and $S 6 \mathrm{k}^{-/-} \mathrm{HSC} / \mathrm{Ps}$ transduced with MLL-AF9 into irradiated recipients. Both recipient groups showed increased wbc count and the presence of donor-derived myeloid cells in the $\mathrm{PB}$ and BM (Supplemental Figure 4, A-F); they also developed AML and had similar median survival times (Supplemental Figure 4G). The frequency and quiescence levels of leukemia stem cell-enriched (LSC-enriched) fractions were also similar in the splenocytes of primary recipients (Supplemental Figure 5, A-C, and ref. 11). To 


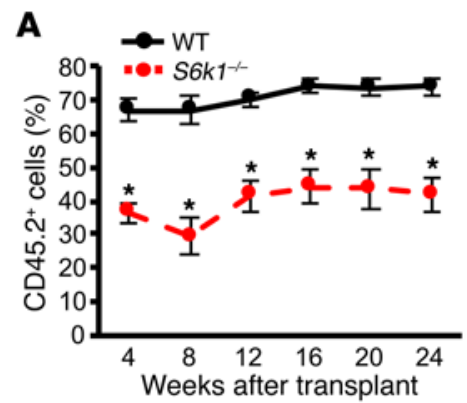

B

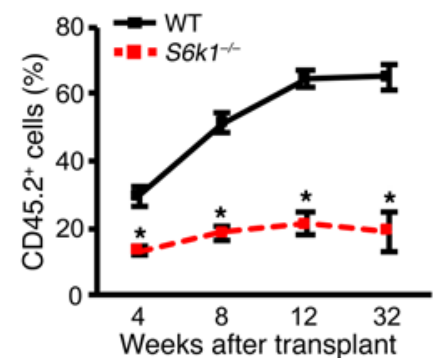

C

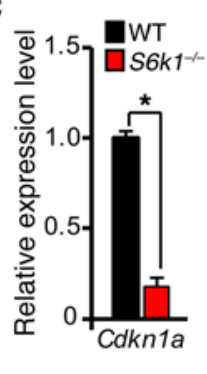

D

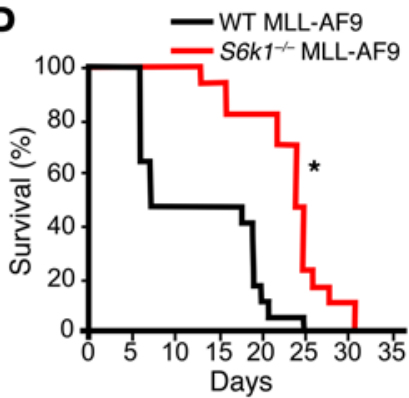

H

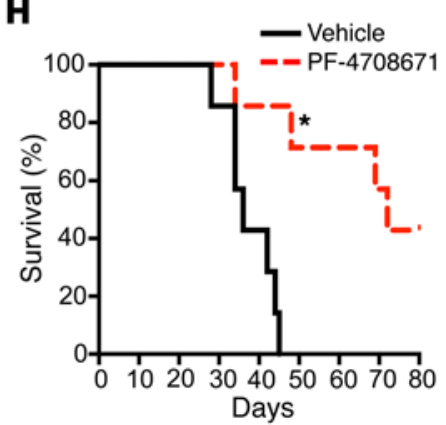

Figure 3. S6K1 is a positive regulator of HSC self-renewal and prolongs the survival of leukemic mice. (A) Quantitative analysis of donor-derived chimerism in PB of secondary recipients of WT and S6k1-- LT-HSCs; mean \pm SEM. ${ }^{*} P<0.001,1$-way ANOVA. $n=9-10 /$ group. (B) Quantitative analysis of donorderived chimerism in PB of tertiary transplant recipients of WT and S6k1-1- LT-HSCs; mean \pm SEM. ${ }^{*} P<0.001,1$-way ANOVA. $n=7-10 /$ group. (C) Expression of Cdkn1a in CD45.2+ LSK cells isolated from WT and S6k1-/- secondary recipients depicted in part A; mean \pm SD. ${ }^{*} P<0.001$. Experiment performed in triplicate. (D) Kaplan-Meier survival curves of secondary recipients of MLL-AFg fusion gene transduced with WT or S6k1//- cells derived from primary recipients. ${ }^{*} P<0.001$ ( $n=17 /$ group). (E) Quantitative representation of Ki-67-negative GFP+ $\mathrm{Cr1}^{-}$Mac1- Sca1-c-Kit ${ }^{+}$cells in BM and spleen of secondary recipients (from part D) of WT or S6k1-- AML cells expressing MLL-AF9; mean \pm SEM. ${ }^{*} P<0.01, t$ test. $n=5$ mice/group. Data representative of 2 independent experiments. (F) Phosphorylation level of AKT and 4E-BP1 in WT and S6k1-/- HSC/Ps expressing MLL-AF9; $n=2$. (G) Phosphorylation level of mTOR and 4E-BP1 in MA9-3 cells following treatment with PF-4708671; $n=3$. (H) Kaplan-Meier survival curves of NOD/SCID mice transplanted with MA9-3 cells treated with either vehicle or PF-4708671. ${ }^{*} P<0.05 . n=7$ /group.

determine the effect of $\mathrm{S} 6 \mathrm{~K} 1$ on the maintenance and propagation of leukemia, we transplanted mice with cells derived from either WT or $S 6 \mathrm{k1}^{-/}$primary recipients who developed leukemia around the same time. Mice transplanted with cells from $\mathrm{S}_{6 \mathrm{k1}}{ }^{--}$primary recipients survived significantly longer than the cohort transplanted with cells derived from WT primary recipients (Figure 3D and Supplemental Figure 6A). The recipients of $S 6 \mathrm{k1}^{-/}$cells depicted in Figure 3D showed significantly reduced spleen weight compared with WT controls (Supplemental Figure 6B). In the same group of mice, the LSC-enriched fraction showed significantly increased quiescence in $\mathrm{S}_{6 \mathrm{k1}} /$ AML cells compared with WT (Figure 3E and Supplemental Figure 6, C-E). To further assess whether S6K1 deficiency affects the LSC fraction, we sorted $\mathrm{Kit}^{+} \mathrm{Grr}^{-}$cells from primary recipients and transplanted them into secondary recipients (2). Recipients of $S 6 \mathrm{kl}^{-/-} \mathrm{Kit}^{+} \mathrm{Gr} 1^{-}$cells expressing MLL-AF9 survived significantly longer compared with WT controls (Supplemental Figure 7A). Furthermore, following tertiary transplant, mice transplanted with cells from $S 6 \mathrm{k1}^{-/-}$secondary recipients from Figure 3D survived significantly longer than the mice transplanted with cells derived from WT secondary recipients (Supplemental Figure 7B). Thus, although S6K1 is not required for the initiation of leukemia, it positively regulates long-term maintenance and leukemia propagation. In an effort to understand the mechanism behind the prolonged survival of mice bearing $S 6 \mathrm{k1}^{-/-}$leukemic cells, we examined the activation of the mTORC1/mTORC2 pathway. Phos- phorylation of AKT and 4E-BP1 was reduced in S6K1-deficient cells expressing MLL-AF9 compared with control (Figure 3F; see complete unedited blots in the supplemental material). To determine whether inhibition of S6K1 activity influences the growth and propagation of human AML cells (MA9-3) (12), we used a specific inhibitor of S6K1 activity, PF-4708671 (13). MA9-3 cells showed a significant reduction in growth with increasing concentrations of PF-4708671 (Supplemental Figure 7C). Inhibition of S6K1 activity also caused reduced phosphorylation of 4E-BP1 and mTOR (Figure 3G; see complete unedited blots in the supplemental material), a common component of mTORC1 and mTORC2, in these cells. Moreover, NOD/SCID mice transplanted with PF-4708671-treated MA9-3 cells showed increased median survival compared with vehicle-treated cells (Figure 3H) Thus, inhibition of S6K1 activity resulted in reduced growth of MLL-AF9-bearing human AML cells both in vivo and in vitro.

Our studies reveal that the self-renewal of HSCs and leukemic potential of these cells are similarly dependent on S6K1. Furthermore, loss of S6K1 in HSCs increases the susceptibility of mice to repeated myelosuppresive stress. Importantly, several biochemical and functional defects observed in the HSC compartment due to S6K1 deficiency resemble those seen in HSCs lacking mTORC1, including reduced BM cellularity and prolonged survival of leukemic mice. Our data also suggest that loss of quiescence in $S 6 \mathrm{k1}^{-/-}$ LT-HSCs could be a result of reduced Cdkn1a levels as S6k1- 
HSCs have properties similar to those of Cdkn1a-deficient HSCs, including reduced quiescence, increased susceptibility to myelotoxic stress, and reduced self-renewal potential (14).

Propagation and relapse of leukemia have been attributed to the presence of LSCs within the leukemic cell population. AKT and $\mathrm{mTORC} 1$ have been shown to be activated in LSCs, rendering them resistant to chemotherapy, which can be reversed by inhibiting AKT activation (15). Previous studies utilizing mTORC1-deficient HSCs expressing MLL-AF9 have shown a similar prolongation in the survival of transplanted mice, which was associated with reduced 4E-BP1 and AKT phosphorylation (2). Prolonged S6K1 loss causes a reduction in mTOR activation, which affects the activity of mTORC1/ mTORC2, resulting in reduced AKT and 4E-BP1 phosphorylation. Previous studies have established a role of glycogen synthase kinase-3 (GSK-3) as a positive regulator of S6K1 activity (16). GSK-3 is a critical regulator of maintenance of stem cell activity in AML cells (17). Inhibition of GSK-3 activity results in downregulation of genes, which maintain LSCs (17). It is possible that S6K1 is one of the downstream substrates of GSK3 in the regulation of maintenance of LSCs. As inhibition of S6K1 activity prolongs the survival of mice bearing human AML cells, it could be used as a potential therapeutic target in the future to treat AML patients.

\section{Methods}

Detailed methods are described in the Supplemental Methods.

Statistics. Statistical differences between 2 groups were determined using the unpaired Student's $t$ test with a 2-tailed distribution. Differences between multiple groups were determined using 1-way
ANOVA followed by Tukey's multiple comparison test. $P<0.05$ was considered significant.

Study approval. All studies were approved by the IACUC of the Indiana University School of Medicine.

\section{Author contributions}

JG conceived, designed, performed, and analyzed experiments and wrote the manuscript. MK and AC performed and analyzed experiments. BR, PM, and RSM performed experiments. NC, YL, and DRP provided reagents. RJC and RK conceived, designed, and analyzed experiments and wrote the manuscript.

\section{Acknowledgments}

We would like to thank James Mulloy (Cincinnati Children's Hospital Medical Center) for providing the MA9-3 cells. We would like to thank Marilyn Wales for providing administrative support. This work was supported in part by grants from the NIH (R01HL077177 to R. Kapur; R01HL075816 to R. Kapur; R01HL081111 to R. Kapur; R01CA173852 to R. Kapur; R01CA134777 to R.J. Chan and R. Kapur; T32HL007910 to A. Chatterjee); the American Cancer Society (PF13-065-01 to A. Chatterjee); and the Riley Children's Foundation.

Address correspondence to: Rebecca J. Chan, 1044 W. Walnut Street, R4-170, Indianapolis, Indiana 46202, USA. Phone: 317.274.4719; E-mail: rchan@iupui.edu. Or to: Reuben Kapur, 1044 W. Walnut Street, R4-168, Indianapolis, Indiana 46202, USA. Phone: 317.274.4658; E-mail: rkapur@iupui.edu.
1. Guo F, et al. Mouse gene targeting reveals an essential role of $\mathrm{mTOR}$ in hematopoietic stem cell engraftment and hematopoiesis. Haematologica. 2013;98(9):1353-1358.

2. Hoshii T, et al. mTORC1 is essential for leukemia propagation but not stem cell self-renewal. JClin Invest. 2012;122(6):2114-2129.

3. Kalaitzidis D, et al. mTOR complex 1 plays critical roles in hematopoiesis and Pten-loss-evoked leukemogenesis. Cell Stem Cell. 2012;11(3):429-439.

4. Sarbassov DD, et al. Prolonged rapamycin treatment inhibits mTORC2 assembly and Akt/PKB. Mol Cell. 2006;22(2):159-168.

5. Chen C, et al. TSC-mTOR maintains quiescence and function of hematopoietic stem cells by repressing mitochondrial biogenesis and reactive oxygen species. J Exp Med. 2008;205(10):2397-2408.

6. Passegue E, Wagers AJ, Giuriato S, Anderson WC, Weissman IL. Global analysis of proliferation and cell cycle gene expression in the regulation of hematopoietic stem and progenitor cell fates.
JExp Med. 2005;202(11):1599-1611.

7. Miyamoto K, et al. Foxo3a is essential for maintenance of the hematopoietic stem cell pool. Cell Stem Cell. 2007;1(1):101-112.

8. Kornblau SM, et al. Simultaneous activation of multiple signal transduction pathways confers poor prognosis in acute myelogenous leukemia. Blood. 2006;108(7):2358-2365.

9. Min YH, et al. Constitutive phosphorylation of Akt/PKB protein in acute myeloid leukemia: its significance as a prognostic variable. Leukemia. 2003;17(5):995-997.

10. Altman JK, et al. Dual mTORC2/mTORC1 target ing results in potent suppressive effects on acute myeloid leukemia (AML) progenitors. Clin Cancer Res. 2011;17(13):4378-4388.

11. Velu CS, et al. Therapeutic antagonists of microRNAs deplete leukemia-initiating cell activity. JClin Invest. 2014;124(1):222-236.

12. Wei J, et al. Microenvironment determines lineage fate in a human model of MLL-AF9 leukemia. Cancer Cell. 2008;13(6):483-495.
13. Pearce LR, et al. Characterization of PF-4708671, a novel and highly specific inhibitor of p70 ribosomal S6 kinase (S6K1). Biochem J. 2010;431(2):245-255.

14. Cheng T, et al. Hematopoietic stem cell quiescence maintained by p21cip1/waf1. Science. 2000;287(5459):1804-1808.

15. Blackburn JS, et al. Clonal evolution enhances leukemia-propagating cell frequency in T cell acute lymphoblastic leukemia through Akt/ mTORC1 pathway activation. Cancer Cell. 2014;25(3):366-378.

16. Shin S, Wolgamott L, Yu Y, Blenis J, Yoon SO. Glycogen synthase kinase (GSK)-3 promotes $\mathrm{p} 70$ ribosomal protein S6 kinase (p70S6K) activity and cell proliferation. Proc Natl Acad Sci US A. 2011;108(47):E1204-E1213.

17. Wang Z, et al. GSK-3 promotes conditional association of CREB and its coactivators with MEIS1 to facilitate HOX-mediated transcription and oncogenesis. Cancer Cell. 2010;17(6):597-608 